\title{
A NOTE ON REGULAR LOCAL NOETHER LATTICES
}

\author{
by JOHNNY A. JOHNSON
}

(Received 17 September, 1973; revised 4 March, 1974)

If $R$ is a local (Noetherian) ring, it is well known that $R$ is regular if and only if its completion is regular. It is the purpose of this note to show that a similar result is true for Noether lattices.

Let $\mathscr{L}$ be a complete lattice on which there is defined a commutative, associative, join distributive (finite and infinite) multiplication such that the unit element of $\mathscr{L}$ is an identity for multiplication. An element $b$ of $\mathscr{L}$ is said to be meet principal if $(c \wedge(d: b)) b=c b \wedge d$, for all $c, d$ in $\mathscr{L} ; b$ is said to be join principal if $d \vee(c: b)=(d b \vee c): b$ for all $c, d$ in $\mathscr{L}$; and $b$ is said to be principal if $b$ is both meet and join principal. $\mathscr{L}$ is called a Noether lattice if $\mathscr{L}$ is modular, satisfies the ascending chain condition, and every element of $\mathscr{L}$ is the join of principal elements. A Noether lattice $\mathscr{L}$ is said to be local if it has a unique maximal (proper) prime element $m$ and we denote this by $(\mathscr{L}, m)$. In general we shall adopt the Noether lattice terminology of $[\mathbf{1}]$.

If $q$ is a $m$-primary element of a local Noether lattice $(\mathscr{L}, m)$, then, for each positive integer $n$, we let $D(q, n)$ denote the lattice dimension of $\mathscr{L} / q^{n}$. We will require the following two known results from [2] (Corollary 3.5, p. 135, and Theorem 3.9, p. 136, respectively).

THEOREM 1. Let $q$ be a m-primary element of $\mathscr{L}$. Then there exists a polynomial $D^{*}(q, x)$ such that $D^{*}(q, n)=D(q, n)$, for all sufficiently large integers $n$.

THEOREM 2. Let $q$ be a m-primary element of $\mathscr{L}$. Then the degree of $D^{*}(q, x)$ is the altitude of $\mathscr{L}$.

Throughout the remainder of this paper, $\mathscr{L}$ will be a local Noether lattice with unique maximal prime element $m$. Elements of $\mathscr{L}$ will be denoted by small letters $a, b, c, \ldots$ As in section 2 of [2] we let $\mathscr{L}^{*}$ be the collection of all formal sums $\sum_{i=1}^{\infty} a_{i}$ of elements of $\mathscr{L}$ such that $a_{i}=a_{i+1} \vee m^{i}$, for $i=1,2, \ldots$. Elements of $\mathscr{L}^{*}$ are denoted by $A, B, C, \ldots$, and for $A$ in $\mathscr{L}^{*}$, let $A=\sum_{i=1}^{\infty} a_{i}$. On $\mathscr{L}^{*}$ define

$$
\begin{gathered}
A \leqq B \quad \text { if and only if } a_{i} \leqq b_{i}, i=1,2, \ldots, \\
A B=\sum_{i=1}^{\infty}\left(a_{i} b_{i} \vee m^{i}\right),
\end{gathered}
$$

so that $\mathscr{L}^{*}$ is a multiplicative lattice which satisfies the ascending chain condition [3, Theorem 2.1, p. 330].

Let $\left\langle e_{i}\right\rangle(i=1,2, \ldots)$ be a sequence of elements of $\mathscr{L}$ such that, for each positive integer $n$,

$$
e_{i+1} \leqq e_{i} \vee m^{n} \text {, for all positive integers } i \geqq n \text {. }
$$


For each positive integer $n$, set $d_{n}=\bigwedge_{i \geq n}\left(e_{i} \vee m^{n}\right)$. Then $D=\sum_{i=1}^{\infty} d_{i}$ is an element of $\mathscr{L}^{*}$, which we will call the element of $\mathscr{L} *$ derived from the sequence $\left\langle e_{i}\right\rangle(i=1,2, \ldots)$. If $a$ is an element of $\mathscr{L}$, set $a^{*}=\sum_{i=1}^{\infty}\left(a \vee m^{i}\right)$, so that $a^{*}$ is the element of $\mathscr{L}^{*}$ derived from the constant sequence $a_{i}=a, i=1,2, \ldots$. For each $A=\sum_{i=1}^{\infty} a_{i}$ in $\mathscr{L}^{*}$, define $C(A)$ in $\mathscr{L}$ by $C(A)=\bigwedge_{i=1}^{\infty} a_{i}$

Remark 1. For each $a$ in $\mathscr{L}$ note that

$$
C\left(a^{*}\right)=C\left(\sum_{i=1}^{\infty}\left(a \vee m^{i}\right)\right)=\bigwedge_{i=1}^{\infty}\left(a \vee m^{i}\right)=a
$$

by $[1$, Corollary 3.2, p. 486$]$.

The following properties of $\mathscr{L}^{*}$ are known and we collect them here for the convenience of the reader (see [3, p. 331] and [4]).

(i) If $\left\langle c_{i}\right\rangle$ is a sequence of principal elements of $\mathscr{L}$ satisfying (1), then the derived element in $\mathscr{L}^{*}$ of $\left\langle c_{i}\right\rangle$ is a principal element of $\mathscr{L}^{*}$.

(ii) $\mathscr{L}^{*}$ is a local Noether lattice with maximal element $m^{*}=\sum_{i=1}^{\infty} m$.

(iii) The map $a \rightarrow a^{*}$ of $\mathscr{L} \rightarrow \mathscr{L}^{*}$ is a multiplicative lattice monomorphism.

(iv) For each natural number $n, \mathscr{L} * / m^{* n} \cong \mathscr{L} / m^{n}$.

The height of a prime element $p$ of a Noether lattice $\mathscr{L}$ is defined to be the supremum of all integers $n$ for which there exists a prime chain $p_{0}<p_{1}<p_{2}<\ldots<p_{n}=p$ in $\mathscr{L}$, and the altitude of $\mathscr{L}$ is defined to be the supremum of the heights of the prime elements of $\mathscr{L}$. A local Noether lattice $\mathscr{L}$ of altitude $k$ is said to be regular in case the unique maximal prime element of $\mathscr{L}$ is the join of $k$ principal elements.

THEOREM 3. $(\mathscr{L}, m)$ is a regular local Noether lattice if and only if $\left(\mathscr{L}^{*}, m^{*}\right)$ is a regular local Noether lattice.

Proof. Since $\mathscr{L}^{*} / \mathrm{m}^{* i} \cong \mathscr{L} / \mathrm{m}^{i}$, for all integers $i \geqq 1$, by (iv), it follows from Theorem 2 that $\mathscr{L}$ and $\mathscr{L}^{*}$ have the same altitude; so let the altitude of $\mathscr{L}$ and $\mathscr{L}^{*}$ be $d$. Assume that $\mathscr{L}^{*}$ is regular and let $a_{1}, a_{2}, \ldots, a_{k}$ be principal elements of $\mathscr{L}$ that form a basis for $m$, so that $m=a_{1} \vee \ldots \vee a_{k}$. Since the altitude of $\mathscr{L}$ is $d$, it follows that $m$ cannot be a join of fewer than $d$ principal elements of $\mathscr{L}$ (cf. [1, Theorem 6.5, p. 496]); thus $k \geqq d$. Since

$$
m^{*}=\left(a_{1} \vee \ldots \vee a_{k}\right)^{*}=a_{1}^{*} \vee \ldots \vee a_{k}^{*}
$$

by (iii), and each $a_{i}^{*}(1 \leqq i \leqq k)$ is principal by (i), it follows that $\left\{a_{1}^{*}, \ldots, a_{k}^{*}\right\}$ forms a basis for the maximal element $m^{*}$ of $\mathscr{L}^{*}$ that can be reduced to a minimal basis that will necessarily have $d$ elements (cf. [2, Theorem 1.4, p. 126]). Thus assume that

$$
m^{*}=a_{i_{1}}^{*} \vee \ldots \vee a_{i_{d}}^{*}
$$


so that

$$
m=C\left(m^{*}\right)=C\left(\left(a_{i_{1}} \vee \ldots \vee a_{i_{d}}\right)^{*}\right)=a_{i_{1}} \vee \ldots \vee a_{i_{d}},
$$

by Remark 1. Consequently, $m$ is the join of $d$ principal elements and therefore $\mathscr{L}$ is regular.

Conversely, if $\mathscr{L}$ is regular, then there are $d$ principal elements $a_{1}, \ldots, a_{d}$ of $\mathscr{L}$ such that

Hence

$$
m=a_{1} \vee \ldots \vee a_{d}
$$

$$
m^{*}=\left(a_{1} \vee \ldots \vee a_{d}\right)^{*}=a_{1}^{*} \vee \ldots \vee a_{d}^{*}
$$

by (iii), where, as above, by (i) each $a_{i}^{*}(1 \leqq i \leqq d)$ is principal in $\mathscr{L}^{*}$, so that $\mathscr{L}^{*}$ is regular. This completes the proof of the theorem.

\section{REFERENCES}

1. R. P. Dilworth, Abstract commutative ideal theory, Pacific J. Math. 12 (1962), 481-498.

2. E. W. Johnson, A-transforms and Hilbert functions on local lattices, Trans. Amer. Math. Soc. 137 (1969), 125-139.

3. E. W. Johnson and J. A. Johnson, $M$-primary elements of a local Noether lattice, Canad. J. Math. 22 (1970), 327-331.

4. E. W. Johnson and J. A. Johnson, $M$-primary elements of a local Noether lattice, Corrigendum, Canad. J. Math. 25 (1973), 448.

UNIVERSITY OF HOUSTON

DBPARTMENT OF MATHEMATICS

Houston, TEXAS 77004

U.S.A. 